\title{
PERFIL LIPÍDICO, GLICÍDICO E PROTEINÚRIA EM PACIENTES COM DIABETES MELLITUS TIPO 2
}

\section{LIPIDIC AND GLICIDIC PROFILE AND PROTEINURIA IN PATIENTS WITH TYPE 2 DIABETES MELLITUS}

\author{
Marisa de Fátima Sauthier, ${ }^{1}$ Rafael de Nogueira Ribeiro, ${ }^{2}$ Bruna Breyer de Freitas, ${ }^{2}$ Aline Marcadenti, ${ }^{4}$ Simara \\ Rufatto Conde ${ }^{1}$ \\ ${ }^{1}$ Univates/Lajeado/Brasil. ${ }^{2}$ Hospital Nossa Senhora da Conceição/Porto Alegre/Brasil. ${ }^{3}$ Universidade Federal de Ciências da Saúde de \\ Porto Alegre/Porto Alegre/Brasil.
}

Autor correspondente: Aline Marcadenti

e-mail: marcadenti.aline@gmail.com

\section{EDITORES}

Thiago Gomes Heck

(Unijuí-Brasil)

Adriane Cristina Bernat Kolankiewicz

(Unijuí-Brasil)

\section{EDITORES DE ÁREA}

Educação \& Saúde

Eva Teresinha de Oliveira Boff

(Unijuí-Brasil)

Fisioterapia \& Saúde

Eliane Roseli Winkelmann

(Unijuí-Brasil)

Ciências Farmacêuticas \& Saúde

Marilei Uecker Pletsch

(Unijuí-Brasil)

\section{Nutrição \& Saúde}

Lígia Beatriz Bento Franz

(Unijuí-Brasil)

Nadia Oliveira

(Unipampa-Brasil)

Ingrid Perry

(UNESC-Brasil)

Enfermagem e suas contribuições para a prática

Adriane Cristina Kolankiewicz

(Unijuí-Brasil)

Crhis de Brum

(UFFS-Brasil)

Neila de Souza

(UFSM-Brasil)

Exercício Físico \& Saúde

Thiago Gomes Heck

(Unijuí-Brasil)

Anderson Zampier Ulbrich

(UFPR)

\section{Editora Unijuí}

Universidade Regional do Noroeste do

Estado do Rio Grande do Sul (Unijuí)

\section{RESUMO}

Objetivo: avaliar o perfil lipídico e glicídico em homens e mulheres com Diabetes Mellitus tipo 2 (DM2) admitidos em um hospital terciário e correlacioná-los com a proteinúria. Método: estudo transversal entre 74 pacientes $\geq 18$ anos com DM2 admitidos em um hospital público de Porto Alegre/RS. Os participantes responderam a um questionário e foram submetidos a medidas de peso, altura e circunferência da cintura. Para avaliação de pacientes acamados ou amputados, o peso e altura foram estimados por meio da circunferência do braço e altura do joelho. Na avaliação bioquímica foram solicitados exames de sódio, potássio, creatinina, ureia, proteinúria, triglicerídeos, hemoglobina glicada, glicemia em jejum, colesterol total, HDL e LDL colesterol. Correlação de Spearman e teste de regressão linear múltipla foram utilizados para correlações e associações. Resultados: no total, 43 homens e 31 mulheres foram avaliados com idade média de 63,28 $\pm 7,46$ anos, 4,51 $\pm 3,25$ anos de estudo e IMC $25,91 \pm 4,19 \mathrm{~kg} / \mathrm{m}^{2}$. Entre os participantes $79,7 \%$ declararam-se brancos e 70,3\% eram aposentados. No gênero masculino, observaram-se maiores valores de colesterol total, HDL colesterol e correlação positiva e significativa entre a proteinúria a e circunferência da cintura $(\mathrm{p}=0,043)$. Valores de HDL colesterol associaram-se negativamente e de forma significativa com a proteinúria independente da idade e da obesidade central entre os homens [Beta -5,1; EP 2,5 (IC 95\% -10,1 a -0,2) P=0,04]. Conclusões: no gênero masculino a proteinúria pode estar associada com HDL colesterol de forma inversa independente de outros fatores.

Palavras-chave: Diabetes Mellitus Tipo 2. Proteinúria. Obesidade abdominal. HDLColesterol.

Submetido em: $5 / 1 / 2015$

Aceito em: 6/10/2016 


\section{Revista}

\section{Contexto}

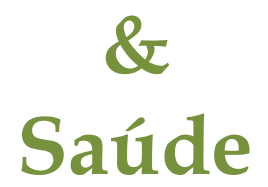

Volume 16

Número 31

2016

ISSN 2176-7114

A Revista Contexto \& Saúde é um periódico do Departamento de Ciências da Vida da Universidade Regional do Noroeste do Estado do Rio Grande do Sul (Unijuí). É um periódico semestral que tem por objetivo a divulgação da produção técnico-científica de temas relacionados à área de Ciências da Saúde.

O escopo da revista abrange a divulgação de resultados de pesquisa que contemplem avanços no processo saúde-doençacuidado e no conhecimento e aplicabilidade de novos processos químicos e biológicos em saúde.

Neste periódico, entende-se que a publicação de estudos com os aspectos epidemiológicos, assistenciais e educacionais em saúde, experimentais e aplicados é uma forma a subsidiar e qualificar a atenção à saúde de modo interdisciplinar.

\begin{abstract}
Objective: To evaluate the lipid and glucose profile in men and women with type 2 diabetes mellitus (DM2) admitted in a tertiary hospital and to correlate them with proteinuria. Method: Cross-sectional study among 74 patients $\geq 18$ years with T2DM admitted in a public hospital in Porto Alegre / RS. Participants answered a questionnaire and weight, height and waist circumference were measured. For evaluation of bedridden patients or amputees, weight and height were estimated by means of the arm circumference and the height of the knee. There were requested biochemical evaluation tests as sodium, potassium, creatinine, urea, proteinuria, triglycerides, glycated hemoglobin, fasting blood glucose, total cholesterol, HDL and LDL cholesterol. Spearman correlation and multiple linear regression were used for correlations and associations. Results: In total, 43 men and 31 women were evaluated with a mean age of $63.28 \pm 7.46$ years, $4.51 \pm 3.25$ years of study and BMI $25.91 \pm 4.19 \mathrm{~kg} / \mathrm{m} 2$. Among all participants $79.7 \%$ declared themselves white and $70.3 \%$ were retired. In men, there were higher values of total cholesterol, HDL cholesterol and a significant positive correlation between proteinuria and waist circumference $(\mathrm{p}=0.043)$. HDL cholesterol levels were associated negatively and significantly with proteinuria independent of age and central obesity among men [Beta -5.1; EP 2.5 (95\% CI: -10.1 to -0.2$) \mathrm{P}=0.04]$. Conclusions: In men, proteinuria may be negatively associated with HDL independent of other factors.
\end{abstract}

Keywords: Diabetes Mellitus, Type 2. Proteinuria. Obesity, Abdominal. Cholesterol, HDL. 


\section{INTRODUÇÃO}

A Diabete mellitus tipo 2 (DM2) é uma síndrome clínica heterogênea caracterizada por anormalidades no metabolismo dos glicídios, lipídeos e proteínas, sendo a elevação no nível glicêmico a de maior repercussão metabólica (ADA, 2014). Essas anormalidades têm como elemento fundamental uma deficiência absoluta ou relativa da função secretora de insulina pelo pâncreas e/ou ação deficiente de insulina nos tecidos-alvos, podendo levar a complicações micro e macrovasculares (SBD, 2009).

A dislipidemia está associada à DM2 (Lu W, 2003) e caracteriza-se por níveis elevados de colesterol total e/ou triglicerídeos e/ou lipoproteínas de baixa densidade (LDL, do inglês low-density lipoprotein) e/ou baixos níveis de lipoproteínas de alta densidade (HDL, do inglês high-density lipoprotein). Hipercolesterolemia é causa direta de eventos cardíacos isquêmicos. Os indivíduos com DM2 já são considerados com alto risco cardiovascular independente de outros fatores de risco, como a alteração dos níveis de lipídeos séricos (XAVIER, 2013).

Uma das principais complicações microvasculares do DM2 é a nefropatia diabética (ND), posto que a proteinúria é uma das primeiras manifestações clínicas indicativas do declínio da função renal nesses pacientes (ADA, 2014). A proteinúria (macro e microalbuminúria) é caracterizada pela perda de proteína pela urina, e sua avaliação é parte importante do diagnóstico, prognóstico e monitoramento de intervenções na ND (ADA, 2014; SBD, 2009). Atualmente, é também apontada como um possível biomarcador de doença cardiovascular (XAVIER, 2013), e indivíduos com essa condição apresentam maior risco para morte (TANIHARA, 2005).

As prevalências de dislipidemia e as baixas taxas de controle entre pacientes com DM2 e proteinúria parecem ser superiores comparativamente aos diabéticos sem perdas urinárias de proteínas (WANG, 2013). O aumento da taxa de excreção urinária de albumina está associado a lipoproteínas ApoB e ao fenótipo de hipertrigliceridemia/hiper-ApoB em indivíduos com DM2 (TSENG, 2005). Ademais, estudos sugerem um potencial papel das dislipidemias (com destaque para baixos valores de HDL colesterol) na patogênese da nefropatia diabética, independente de outros fatores (MARCOVECCHIO, 2009; BULUM et al., 2011, 2013). Grande parte dos estudos que avaliaram a associação entre dislipidemias e a presença de proteinúria, entretanto, foram desenvolvidos em pacientes com Diabete mellitus tipo 1 (DM1) (MARCOVECCHIO, 2009; BULUM et al., 2011, 2013).

Considerando a prevalência elevada de dislipidemias em pacientes diabéticos, e que, associada à proteinúria, parece contribuir para um pior prognóstico nesses indivíduos, o objetivo do presente estudo foi avaliar o perfil lipídico e glicídico em homens e mulheres com DM2 admitidos em um hospital terciário, e correlacioná-los com a proteinúria.

\section{METODOLOGIA}

Trata-se de um estudo transversal, realizado em um hospital público de Porto Alegre/RS, de maio a outubro de 2014. A amostragem foi por conveniência, composta por 74 pacientes admitidos na Unidade de Cirurgia Vascular do Hospital Nossa Senhora da Conceição (HNSC). Foram incluídos indivíduos adultos, com idade entre 35 e 80 anos e diagnóstico clínico de DM2, que aceitaram participar da pesquisa assinando o Termo de Consentimento Livre e Esclarecido (TCLE). Foram excluídos os pacientes com DM1 que não apresentaram condições cognitivas para preenchimento do questionário ou com amputações que impossibilitassem a verificação do peso e da altura. O estudo foi aprovado pelos Comitês de Ética em Pesquisa do Centro Universitário Univates (Parecer $n^{\circ} 537.463$ ) e do HNSC (Protocolo no 14-039). 
Os pacientes responderam a um questionário padronizado com variáveis demográficas e socioeconômicas como idade, cor da pele autorreferida, ocupação, escolaridade, tabagismo (fumante, ex-fumante, nunca fumou), consumo de bebidas alcoólicas (sim, já consumiu, não) e prática de atividades físicas (sim/não).

A avaliação antropométrica foi realizada em balança antropométrica, da marca Filizola®, com capacidade máxima de $200 \mathrm{~kg}$ e mínima de $1 \mathrm{~kg}$, com graduação a cada $0,050 \mathrm{~g}$ e régua para altura até $2 \mathrm{~m}$. Os indivíduos foram pesados e medidos em pé, no centro da base da balança, eretos, com os pés juntos, braços estendidos ao longo do corpo, sem sapatos e com roupa leve. Já para os pacientes sem condições de aferição de peso, o mesmo foi estimado pela equação de Chumlea (1988). O índice de massa corporal (IMC) foi calculado a partir da fórmula: peso atual $(\mathrm{kg}) /$ altura $\left(\mathrm{m}^{2}\right)$ e classificados adultos segundo a OMS (WORLD, 2000) e para idosos (LIPSCHITZ, 1994). A circunferência da cintura $(\mathrm{em} \mathrm{cm})$ foi aferida utilizando uma fita métrica inextensível e flexível, com o indivíduo em pé, ao final da expiração, circundando a região abdominal no ponto médio entre a crista ilíaca e o rebordo costal inferior (LOHMAN; ROCHE; MARTORELL, 1988).

Dados referentes à pressão arterial sistólica (PAS, em mm $\mathrm{Hg}$ ) e diastólica (PAD, em mmHg) foram obtidos a partir de prontuário médico, sendo registrada a primeira aferição do dia. Exames bioquímicos foram conseguidos também por meio de registros em prontuário eletrônico, e analisados pelo Laboratório de Análises Clínicas do HNSC a partir dos seguintes pontos de corte: sódio (135 a $145 \mathrm{mEq} / \mathrm{l})$, potássio (3,5 a 5,0 mEq/l), creatinina $(0,4$ a $1,2 \mathrm{mg} / \mathrm{dl})$, ureia $(10$ a $50 \mathrm{mg} / \mathrm{dl})$, proteinúria $(<0,15 \mathrm{~g} / 24 \mathrm{hrs})$, triglicerídeos $(<150 \mathrm{mg} / \mathrm{dl})$, hemoglobina glicada $(<7 \%)$, glicemia em jejum $(70$ a $99 \mathrm{mg} / \mathrm{dl})$, colesterol total $(<200 \mathrm{mg} / \mathrm{dl})$, HDL colesterol $(>60 \mathrm{mg} / \mathrm{dl})$ e LDL colesterol $(<100 \mathrm{mg} / \mathrm{dl})$.

Os dados foram digitados em uma planilha do tipo Excel®. Variáveis contínuas foram descritas como média e desvio-padrão, e as categóricas como números absolutos e proporções. Comparações entre médias foram feitas utilizando-se os testes t de Student e de Mann-Whitney, e proporções foram comparadas por meio do teste Quiquadrado de Pearson. Correlações foram obtidas por intermédio de correlações de Spearman, e regressão linear múltipla foi utilizada no teste de associações. Considerou-se um nível de significância de 5\%, e as análises foram realizadas pelo programa estatístico Software Statistical Package for the Social Sciences (SPSS®) versão 17 para Windows.

\section{RESULTADOS}

No total, 43 homens e 31 mulheres foram avaliados com idade média de 63,28 $\pm 7,46$ anos, 4,51 $\pm 3,25$ anos de estudo e IMC $25,91 \pm 4,19 \mathrm{~kg} / \mathrm{m}^{2}$. Entre os participantes, $79,7 \%$ declararam-se brancos e $70,3 \%$ eram aposentados.

A Tabela 1 mostra as características dos participantes de acordo com o gênero. Comparativamente às mulheres, os homens relataram ser fumantes ou ex-fumantes, consumir ou já ter consumido bebidas alcoólicas com maior frequência e não praticar atividades físicas, sendo apenas o consumo de álcool estatisticamente significativo $(\mathrm{p}=$ 0,003). Observaram-se maiores valores de circunferência da cintura, IMC e pressão arterial também entre os homens, mas sem diferença significativa entre os gêneros. Em relação aos exames bioquímicos, apenas os valores de colesterol total e HDL colesterol diferenciaram-se significativamente entre homens e mulheres, considerando que no gênero masculino os valores encontrados foram maiores $(\mathrm{p}=0,04 \mathrm{e} p<0,001$ respectivamente). 
Tabela 1 - Características dos 74 participantes de acordo com o gênero [média \pm DP, n(\%)]. Porto Alegre, RS

\begin{tabular}{|c|c|c|c|}
\hline & Homens $(n=43)$ & Mulheres $(n=31)$ & Valor-P \\
\hline Idade (anos) & $62,99 \pm 6,77$ & $63,67 \pm 8,41$ & $0,70^{*}$ \\
\hline Escolaridade (anos) & $5,16 \pm 3,96$ & $4,05 \pm 2,57$ & $0,18 *$ \\
\hline Tabagismo & & & $0,44 \dagger$ \\
\hline Fuma & $7(77,8)$ & $2(22,2)$ & \\
\hline Ex-fumante & $28(54,9)$ & $23(45,1)$ & \\
\hline Nunca fumou & $6(42,9)$ & $8(57,1)$ & \\
\hline Consumo de álcool & & & $0,003 \dagger$ \\
\hline Sim & $1(100)$ & $0(0,0)$ & \\
\hline Já consumiu & $39(67,2)$ & $19(32,8)$ & \\
\hline Não & $3(20,0)$ & $12(80,0)$ & \\
\hline Atividade física & & & $0,47 \dagger$ \\
\hline Sim & $0(0,0)$ & $1(100)$ & \\
\hline Já praticou & $7(63,6)$ & $4(36,4)$ & \\
\hline Não & $36(58,1)$ & $26(41,9)$ & \\
\hline Circunferência da cintura, em cm & $100,44 \pm 10,8997$ & $97,23 \pm 11,97$ & $0,23 *$ \\
\hline Índice de massa corporal (IMC), em $\mathrm{kg} / \mathrm{m}^{2}$ & $26,10 \pm 4,21$ & $25,64 \pm 4,21$ & $0,65 *$ \\
\hline Pressão arterial sistólica (PAS), em mmHg & $130,65 \pm 20,32$ & $126,28 \pm 13,63$ & $0,27 *$ \\
\hline Pressão arterial diastólica (PAD), em mmHg & $80,00 \pm 11,25$ & $77,91 \pm 10,13$ & $0,41 *$ \\
\hline Sódio (mEq/l) & $135,97 \pm 4,55$ & $136,00 \pm 4,31$ & $0,98 *$ \\
\hline Potássio (mEq/1) & $4,53 \pm 0,77$ & $4,56 \pm 0,58$ & $0,87 *$ \\
\hline Creatinina $(\mathrm{mg} / \mathrm{dl})$ & $1,02 \pm 0,39$ & $1,13 \pm 0,56$ & $0,34^{*}$ \\
\hline Ureia (mg/dl) & $42,94 \pm 20,70$ & $43,65 \pm 21,24$ & $0,89 *$ \\
\hline Colesterol total (mg/dl) & $164,94 \pm 49,88$ & $143,63 \pm 37,66$ & $0,04 *$ \\
\hline HDL (mg/dl) & $45,06 \pm 15,03$ & $29,77 \pm 16,50$ & $<0,001^{\ddagger}$ \\
\hline $\mathrm{LDL}(\mathrm{mg} / \mathrm{dl})$ & $83,32 \pm 39,32$ & $76,16 \pm 30,94$ & $0,38 *$ \\
\hline Triglicerídeos (mg/dl) & $184,94 \pm 81,29$ & $182,51 \pm 77,41$ & $0,89 *$ \\
\hline Glicemia de jejum (mg/dl) & $187,97 \pm 82,66$ & $167,19 \pm 69,02$ & $0,24 *$ \\
\hline Hemoglobina glicada $(\%)$ & $9,18 \pm 2,39$ & $10,16 \pm 10,23$ & $0,60 *$ \\
\hline Proteinúria (g/24hrs) & $0,50 \pm 1,02$ & $0,32 \pm 0,48$ & $0,73^{\ddagger}$ \\
\hline
\end{tabular}

$\dagger$ Teste Qui-quadrado de Pearson $\quad$ † Teste Mann-Whitney * Teste t de Student 
Com relação às variáveis antropométricas, a circunferência da cintura correlacionou-se positivamente com proteinúria nos homens $(\mathrm{rho}=0,31 ; \mathrm{P}=0,04)$, mas não entre as mulheres $(\mathrm{rho}=-0,04 ; \mathrm{P}=0,8)$. $\mathrm{O} \mathrm{IMC}$ não apresentou correlação significativa com a proteinúria em homens e mulheres.

A Tabela 2 mostra as correlações entre proteinúria e variáveis bioquímicas nos gêneros masculino e feminino. Valores de HDL colesterol associaram-se negativa e significativamente com a proteinúria nos homens $(\mathrm{rho}=-$ 0,33; $\mathrm{P}=0,03$ ); nas mulheres, não houve correlações significativas. Após ajuste para a idade e circunferência da cintura, níveis de proteinúria permaneceram inversamente associados com HDL colesterol entre os homens [Beta -5,1; EP 2,5 (IC 95\% -10,1 a -0,2) P= 0,04]. Não foram observadas correlações entre o perfil glicídico e proteinúria entre os pacientes avaliados.

Tabela 2 - Correlação* (rho) entre proteinúria e variáveis bioquímicas nos gêneros masculino e feminino. Porto Alegre, RS

\begin{tabular}{lcc}
\hline Variáveis bioquímicas & $\begin{array}{c}\text { Homens } \\
(\mathbf{n = 4 3})\end{array}$ & $\begin{array}{c}\text { Mulheres } \\
(\mathbf{n}=\mathbf{3 1})\end{array}$ \\
\hline PAS (mmHg) & 0,01 & 0,28 \\
PAD (mmHg) & 0,09 & 0,10 \\
Sódio (mEq/l) & $-0,12$ & $-0,23$ \\
Potássio (mEq/l) & 0,01 & 0,28 \\
Creatinina (mg/dl) & 0,22 & 0,11 \\
Uréia (mg/dl) & 0,27 & 0,34 \\
Colesterol total (mg/dl) & 0,15 & $-0,01$ \\
HDL (mg/dl) & $-0,33^{\ddagger}$ & 0,17 \\
LDL (mg/dl) & 0,19 & 0,01 \\
Triglicerídeos (mg/dl) & 0,17 & $-0,08$ \\
Glicemia de jejum (mg/dl) & $-0,05$ & $-0,04$ \\
Hemoglobina glicada (\%) & 0,05 & $-0,12$ \\
\hline \multicolumn{2}{c}{$*$ Correlação de Spearman } & $\$ P<0,05$
\end{tabular}

\section{DISCUSSÃO}

O presente estudo concluiu que no gênero masculino a proteinúria pode estar associada com o HDL colesterol de forma inversa, independente da idade e da obesidade abdominal. Entre as mulheres não foram encontradas correlações entre o perfil lipídico e proteinúria, e não foram detectadas associações entre perfil glicídico e proteinúria na amostra avaliada.

A caracterização da amostra deste estudo em relação à média de idade em ambos os gêneros foi de aproximadamente 64 anos, resultado semelhante ao estudo de Batista et al. (2005) no qual a maior parte dos diabéticos encontrava-se na faixa etária de 50 a 69 anos $(63,5 \%)$. 
O IMC médio da população avaliada foi $25,91 \mathrm{~kg} / \mathrm{m}^{2}$. Para Batista et al. (2002), a alta prevalência de excesso de peso corrobora com os estudos que indicam a presença da adiposidade elevada como um fator de risco para DM2. Os valores médios de IMC, mais elevados entre os homens, estão de acordo com os resultados de estudos de avaliação nutricional realizados em diversas regiões do Brasil, os quais demonstram maiores prevalências de excesso de peso entre os homens (VIGITEL, 2012).

Daniele et al. (2013) demonstraram em seu estudo que os pacientes diabéticos tipo 2 são, em sua maioria, sedentários. No atual estudo, apenas um participante praticava atividade física. Os benefícios proporcionados pela prática de exercício físico no indivíduo diabético e que geralmente é hipertenso, podem ser agudos (em curto prazo) ou crônicos (longo prazo) (OLIVEIRA, et al., 2003), e a prática regular de exercícios físicos por eles, dentro das intensidades recomendadas, podem resultar em redução de $10 \%$ a $20 \%$ na hemoglobina glicada, e também em melhor transporte de oxigênio pela corrente sanguínea (ADA, 2005).

Vazquez et al. (2007) demonstraram correlações positivas entre circunferência da cintura e os parâmetros bioquímicos, com destaque para a glicemia de jejum e triglicerídeos em pacientes do gênero feminino. No presente estudo houve correlação positiva e significativa entre proteinúria e circunferência da cintura entre o gênero masculino: sabe-se que uma das limitações do IMC é a incapacidade de discriminar compartimentos corporais e que homens apresentam maiores proporções de massa magra quando comparados às mulheres. Dessa forma, a circunferência da cintura nesses indivíduos parece ter refletido de forma mais adequada os efeitos da adiposidade sobre a proteinúria.

A inibição da ação da insulina leva ao desenvolvimento de resistência à insulina e do DM2, podendo ser ocasionada pela adiposidade central, fator clássico para o aparecimento da resistência à insulina e associada ao aumento dos níveis de triglicérides e baixas concentrações de HDL colesterol, aumentando o risco para disfunções macrovasculares (ARSA et al., 2009; SAVAGE, et al., 2005). No presente estudo observou-se correlação negativa entre proteinúria e o colesterol HDL no gênero masculino independente da obesidade central, confirmando os achados de outros pesquisadores acerca do papel dos baixos valores de HDL colesterol na gênese da nefropatia diabética (MARCOVECCHIO et al., 2009; BULUM et al., 2011, 2013).

Estudos que avaliaram associações entre albuminúria e perfil lipídico em pacientes com DM2 detectaram resultados semelhantes ao demonstrado no presente estudo (TSENG, PAN, 2012), quando valores de HDL colesterol foram significativamente inferiores entre os pacientes com presença de proteínas na urina. Esses estudos, porém, avaliaram indivíduos orientais, e é importante destacar as diferenças principalmente no que se refere à composição corporal diferenciada nessas populações.

Entre as limitações do estudo estão a falta de informações acerca dos hábitos alimentares desses indivíduos assim como do uso de medicamentos - que poderiam ter influenciado nos valores dos exames bioquímicos. Além disso, o delineamento transversal não é capaz de indicar um risco real para o desenvolvimento da nefropatia a partir de valores alterados de lipídeos séricos, em razão da falta de seguimento dos pacientes.

\section{CONCLUSÃO}

Conclui-se que a proteinúria pode estar associada negativamente com o HDL colesterol independente da idade e da obesidade abdominal entre os homens, fatores de risco importantes para a gênese do DM2 e de suas complicações. Entre as mulheres não foram encontradas correlações entre o perfil lipídico e a proteinúria, e não foram detectadas associações entre perfil glicídico e proteinúria na amostra em geral. 
Sugere-se que esses resultados sejam replicados em outros pacientes com DM2 a fim de confirmar esses resultados na população brasileira, e que nesses pacientes a avaliação da proteinúria e do HDL colesterol de forma concomitante seria útil para a detecção precoce de risco elevado para complicações cardiovasculares.

\section{REFERÊNCIAS}

AMERICAN DIABETES ASSOCIATION (ADA). Standards of Medical Care in Diabetes - 2014. Diabetes Care, v. 37 (Supplement 1):S14-S80, 2014.

AMERICAN DIABETES ASSOCIATION (ADA). Standards of Medical Care in Diabetes. Diabetes Care, v. 28, S37-S42, 2005.

ARSA, G. et al. Diabetes Mellitus tipo 2: aspectos fisiológicos, genéticos e formas de exercício físico para seu controle. Rev Bras Cineantropom Desempenho Hum, v. 11, n. 1, p. 103-111, 2009.

BATISTA, M. C. R. et al. Avaliação de indicadores antropométricos de adultos e idosos brasileiros. Nutrire: Rev Soc Bras Alim Nutr., v. 23, p.67-78, 2002.

- Avaliação dos resultados da atenção multiprofissional sobre o controle glicêmico, perfil lipídico e estado nutricional de diabéticos atendidos em nível primário. Rev. Nutr., Campinas, n. 18, v. 2, p. 2-9, 2005.

BULUM, T. et al. Lower levels of HDL2 cholesterol are associated with microalbuminuria in patients with type 1 diabetes. Acta Med Croatica, v. 65, n. 3, p. 243-50, 2011.

Lower levels of total HDL and HDL3 cholesterol are associated with albuminuria in normoalbuminuric Type 1 diabetic patients. J Endocrinol Invest, v. 36, n. 8, p. 574-578, 2013.

CHUMLEA, C. et al. Prediction of body weight for the nonambulatory elderly from anthropometry. $J$ Am Diet Assoc, n. 88, v. 5, p. 564-69, 1988.

DANIELE, T. M. et al. Associations among physical activity, comorbidities, depressive symptoms and health related quality of life in type 2 diabetes. Arq Bras Endocrinol Metabol, v. 57, n. 1, p .44-50, 2013.

LIPSCHITZ, D. A. Screening for nutritional status in the elderly. Primary Care, n. 21, v. 1, p. 55-67, 1994.

LOHMAN, T. G.; ROCHE, A. F. \& MARTORELL, R. Anthropometric Standardization Reference Manual. Champaign, Illinois: Human Kinetics. 1988.

LU, W. et al. Non-HDL cholesterol as a predictor of cardiovascular disease in type 2 diabetes: the strong heart study. Diabetes Care, v. 26, n. 1, p. 16-23, 2003.

MARCOVECCHIO. M. L. et al. Prevalence of abnormal lipid profiles and the relationship with the development of microalbuminuria in adolescents with type 1 diabetes. Diabetes Care Hypertension, v. 32, n. 4, p. 658-63, 2009.

OLIVEIRA, E. M. et al. Sistema renina-angiotensina: interação gene-exercício. Rev Bras Hipertens, v. 10, n. 2, p. 125-129, 2003.

PAN, J. et al. Non-high-density lipoprotein cholesterol is associated more closely with albuminuria in Chinese type 2 diabetic patients with normal renal function, compared with traditional lipid parameters. J Clin Lipidol, v. 6, n. 4, p. 382-387, 2012.

SAVAGE, D. B. et al. Mechanisms of insulin resistance in humans and possible links with inflammation. Hypertension, v. 45, n. 5, p. 828-833, 2005.

SOCIEDADE BRASILEIRA DE DIABETES. SBD. Diretrizes SBD 2009. 3.ed. Itapevi, SP: A. Araújo Silva Farmacêutica, 2009. 400 p.

TANIHARA, S. et al. Proteinuria is a prognostic marker for cardiovascular mortality: NIPPON DATA 80, 1980-1999. J Epidemiol, v. 15, p. 146-153, 2005. 
TSENG, C. H. Lipid abnormalities associated with urinary albumin excretion rate in Taiwanese type 2 diabetic patients. Kidney Int, v. 67 , n. 4, p. 547-553, 2005.

VAZQUEZ, G. et al. Comparison of body mass index, waist circumference, and waist/hip ratio in predicting incident diabetes: a metaanalysis. Epidemiol Rev., v. 29, p. 115-128, 2007.

VIGITEL Brasil 2011. Vigilância de fatores de risco e proteção para doenças crônicas por inquérito telefônico. Brasília: Ministério da Saúde; Secretaria de Gestão Estratégica e Participativa, 2012.

WANG, S. H. et al. Prevalence and control of dyslipidaemia among diabetic patients with microalbuminuria in a Chinese hospital. Diab Vasc Dis Res , v. 10, n. 2, p. 169-78, 2013.

WORLD HEALTH ORGANIZATION. WHO. Obesity: preventing and managing the global epidemic. Report of a WHO Consultation. Geneva: World Health Organization, 2000.

XAVIER, H. T. et al. V Diretriz Brasileira de Dislipidemias e Prevenção da Aterosclerose. Arq Bras Cardiol, v. 101, (4Supl.1):1-22, 2013. 